\title{
An overview of applications of CRISPR-Cas technologies in biomedical engineering
}

\author{
Saleh Jamehdor ${ }^{1,2,3 *}$, Kasra Arbabi Zaboli4*, Sina Naserian ${ }^{5,6}$, Jose Thekkiniath ${ }^{7}$, \\ Honey Alef Omidy ${ }^{8}$, Ali Teimoori ${ }^{1}$, Behrooz Johari ${ }^{4}$, Amir Hossein Taromchi ${ }^{4}$, \\ Yu Sasano ${ }^{9}$, Saeed Kaboli ${ }^{4}$
}

${ }^{1}$ Department of Virology, Faculty of Medicine, Hamadan University of Medical Sciences, Hamadan, Iran ${ }^{2}$ Department of Molecular Medicine, Institute of Medical Biotechnology, National Institute of Genetic Engineering and Biotechnology, Tehran, Iran

${ }^{3}$ Department of Biology, Faculty of Sciences, University of Sistan and Baluchestan, Zahedan, Iran ${ }^{4}$ Department of Medical Biotechnology, School of Medicine, Zanjan University of Medical Sciences, Zanjan, Iran

${ }^{5}$ INSERM UMR-S-MD 1197/Ministry of the Armed Forces, Biomedical Research Institute of the Armed Forces (IRBA), Paul-Brousse Hospital Villejuif, and CTSA Clamart, France

${ }^{6}$ SivanCell, Sivan Aryo Pharmed, Tehran, Iran

${ }^{7}$ Fuller Laboratories, Fullerton, CA, USA

${ }^{8}$ Department of Biochemistry and Molecular Biology, University of Southern California, Los Angeles, CA, USA

${ }^{9}$ Department of Applied Microbial Technology, Faculty of Biotechnology and Life Science, Sojo University, Kumamoto, Japan

*Saleh Jamehdor and Kasra Arbabi Zaboli equally contributed to work

\begin{abstract}
Clustered Regulatory Interspaced Short Palindromic Repeats (CRISPR) is one of the major genome editing systems and allows changing DNA levels of an organism. Among several CRISPR categories, the CRISPR-Cas9 system has shown a remarkable progression rate over its lifetime. Recently, other tools including CRISPR-Cas12 and CRISPR-Cas13 have been introduced. CRISPR-Cas9 system has played a key role in the industrial cell factory's production and improved our understanding of genome function. Additionally, this system has been used as one of the major genome editing systems for the diagnosis and treatment of several infectious and non-infectious diseases. In this review, we discuss CRISPR biology, its versatility, and its application in biomedical engineering. (Folia Histochemica et Cytobiologica 2020, Vol. 58, No. 3, 163-173)
\end{abstract}

Key words: Genome editing; sgRNA; CRISPR-Cas9; CRISPR-Cas12; CRISPR-Cas13

Abbreviations: AAV — adeno-associated virus; ADAR adenosine deaminases acting on RNA; Cas9 - CRISPR-

Correspondence address: Saeed Kaboli

Department of Medical Biotechnology,

School of Medicine, Zanjan University

of Medical Sciences, Zanjan, Iran

e-mail: kaboli2017@zums.ac.ir

Yu Sasano,

Department of Applied Microbial Technology,

Faculty of Biotechnology and Life Science,

Sojo University, Kumamoto, Japan

e-mail: sasano@bio.sojo-u.ac.jp -associated endonuclease 9; saCas9 — Cas9 from Staphylococcus aureus; spCas9 - Cas9 from Streptococcus pyogenes; HDR - homology-directed repair; $\mathrm{HNH}$ - an endonuclease domain named for characteristic histidine and asparagine residues; HPRT — hypoxanthine phosphoribosyltransferase; Indel — insertion and/or deletion; MHC - Major Histocompatibility Complex; NAG - Nucleotide-Adenine-Guanine; NGG - Nucleotide-Guanine-Guanine; NHEJ — non-homologous end-joining; PAM — Protospacer Adjacent Motifs; RuvC - an endonuclease domain named for an $E$. coli protein involved in DNA repair 


\section{Introduction}

CRISPR-Cas (Clustered Regularly Interspaced Short Palindromic Repeats-CRISPR associated Cas enzyme) is an advanced adaptive immune system in bacteria and most archaea which protects them through the degradation of invasive DNA [1]. The history of the exploration of the CRISPR-Cas system turns back to 1987 when Ishino and co-workers at Osaka University reported repeated DNA fragments in cluster form in the Escherichia coli genome [2]. While they were attempting to investigate the alkaline phosphatase isozyme encoding gene, a special repetitive sequence was observed downstream of the gene. This repetitive sequence was composed of five completely similar repetitive 29 nucleotides sequences that are dispersed by 32 nucleotide unique sequences [2]. In the following years, similar repetitive sequences were found in other $E$. coli strains and in enterobacteria that are closely related to E.coli as well as in Shigella dysenteria [3]. Several 36-base pair (bp) direct repeats interspaced by 35-46 bp unparalleled spacers were reported in Mycobacterium tuberculosis [4]. The repetitive sequence of the CRISPR in archaea was discovered in 1993, while Mojica and co-workers were investigating the salinity effects on the growth of Haloferax mediterranei. These researchers observed a long DNA sequence in the genome of these archaea which composed of regulatory repeats, although there was no similarity between these sequences and E.coli repeats [5]. The function of these repeats remained unclear for almost 20 years after its discovery. Several names including multiple direct repeats (DRs) [6], short regulatory spaced repeats (SRSR) [7], and large clusters of tandem repeats (LCTR) [8] have been suggested.

The term CRISPR was coined by Jansen and coworkers [9] and became accepted by researchers as this acronym reflects the structural features of repeats. In this review, we summarize CRISPR-Cas mechanisms and its applications in biotechnology and biomedical engineering. We propose insights into our understanding of CRISPR-Cas and discuss the open questions in the field.

\section{Molecular mechanisms of CRISPR-Cas system}

In the CRISPR-Cas system, RNA harboring the spacer sequence supports the CRISPR-associated (Cas) proteins to recognize and cleave foreign pathogenic DNA which is followed by cell repairing. There have been two repairing mechanisms discovered; non-homologous end-joining (NHEJ) (with more probability) and homology-directed repair (HDR) mechanisms. To knock-out a specific gene by the CRISPR system, NHEJ has been used (cells naturally use this mechanism with a high percentage to escape death after a double-strand break). On the other hand, HDR has been used for knock-in and occurs at a low percentage (Fig. 1). For HDR knock-in of any DNA fragments, either a single-stranded oligodeoxynucleotide (ssODN) or a double-stranded donor plasmid (dsODN) is used as a template in combination with nucleases to achieve accurately targeted and efficient insertion into the target DNA site. Previous studies have shown that ssODN-mediated knock-in is more efficient as compared to double-stranded plasmid-mediated knock-in [10-12].

CRISPR-Cas system consists of a crRNA (CRISPR RNA) complemented with targeted DNA which causes the attachment of crRNA to this DNA and is involved in recognition. RNA that is attached to the 3 ' end of crRNA is called tracrRNA (trans-activating CRISPR RNA) which transcribes from the same cluster of the CRISPR locus. For recognition and double-stranded DNA cleaving, annealing of these two RNAs to create a single guide RNA (sgRNA) and subsequent attachment of sgRNA to Cas9 protein is required $[1,13]$. One of the major sequences called Protospacer Adjacent Motif (PAM) is also involved in recognition, while the length and number of this sequence may vary in different bacterial systems. This sequence is located close to the $\sim 20$ nucleotides sequence which is recognized by crRNA. The stemloops formed at 3' end of tracrRNA are critical for the function of CRISPR $[14,15]$.

\section{Types of CRISPR systems}

Based on CRISPR/Cas loci, CRISPR systems have been classified into six types (I-VI) and all of them contain a distinctive Cas nuclease along the interference stage [1]. Of these, type I and III systems use a large multi-Cas protein complex to promote the target sequence cleavage, whereas the type II system utilizes a single nuclease called Cas9 [15, 16]. Cas12 and Cas 13 are nuclease proteins belonging to type $\mathrm{V}$ and VI, respectively [17]. In this review, we focus on three important types of CRISPR/Cas systems.

\section{CRISPR-Cas9}

Among the several types of CRISPR-Cas systems, CRISPR-Cas9 has been most commonly used and is derived from Streptococcus pyogenes. CRISPR-Cas9 system has been harnessed to mediate genome editing in several cell types and can be exploited to generate gene knock-outs (via insertion/deletion) or knockins (via the HDR). PAM sequence of this bacterium 


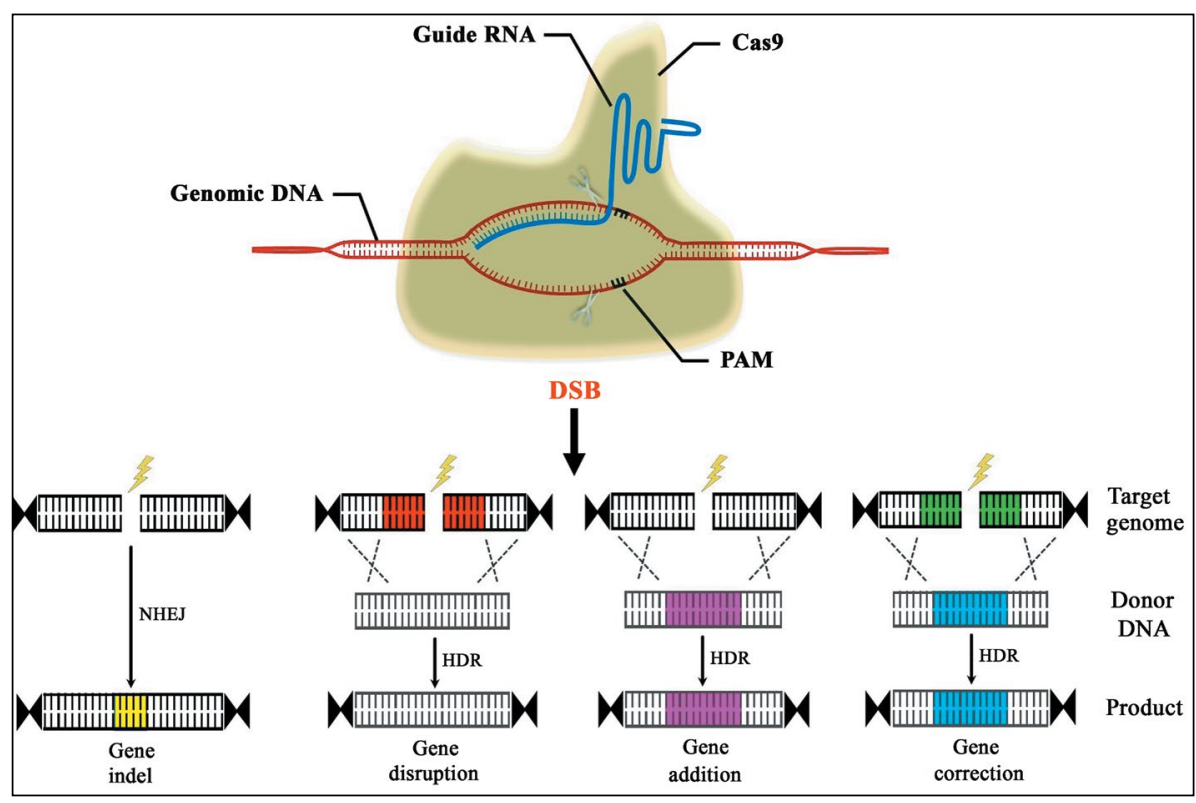

Figure 1. Illustration of the CRISPR-Cas9 mediated gene editing. The CRISPR/Cas9 system which has been functioned in different organisms has a sgRNA consisting of a backbone with three stem-loops and a complementary sequence with a 20-nucleotide target (shown in blue color). It also has a PAM (shown in black color) that in $80 \%$ of cases is NGG and in $20 \%$ of cases is NAG. After binding of 20-nucleotide to the target DNA, endonuclease distinguishes it and cuts three nucleotides after the PAM as blunt end using its endonuclease property (Some studies showed that Cas9 is first attached to sgRNA, and then the target DNA is detected). Afterward, the cell uses an NHEJ repair method to add or reduce some nucleotides (gene indel, shown in yellow color). This method is used to knock out genes. If the cell uses HDR repair, it uses a DNA similar to the cut-off region as a template and performs repair. This type of repair is used to create knock-in in the cell and destroy, add, and correct the gene (shown in no color, pink, and blue respectively).

is 80 percent NGG and could be 20 percent NAG, while other CRISPR systems may contain different PAM sequences [14]. Bioinformatics tools have been used to link tracrRNA to crRNA resulting in a single unique RNA that can be used in genome editing. Several vectors have been generated to exploit the potentials of CRISPR-Cas9 technology [18, 19]. Molecular studies showed that Cas9 endonuclease has two domains (RuvC and $\mathrm{HNH}$ ) on Cas9 protein and each domain cut one strand in a double-stranded DNA. While D10 (aspartic acid 10) that exists on the RuvC domain is involved in cutting the non-complementary strand with sgRNA, H840 (histidine 840) located on the $\mathrm{HNH}$ domain is required for cutting the complementary strand with sgRNA. To create a Cas9 that only connects to the guided location without applying a double-stranded break (Dead Cas9 or dCas9), both nuclease domains need to be mutated [20,21] (Fig. 2). On the other hand, effector domains could be connected to carboxyl-terminal of the dCas9 protein that may result in activation of gene expression by transcriptional activators such as VP64 (a transcription activator protein from Herpes Simplex Virus), inhibition of gene expression by KRAB effector (Krüppel associated box domain), epigenetic changes (KDM [histone lysine demethylase] or HAT/HDAC [histone acetyltransferase/ /histone deacetylase]), tracking of DNA (often with GFP [green fluorescent protein]) and specific DNA isolation by affinity tag (and base-changer deaminase domain) [22-25]. One such effector is adenosine deaminase enzyme (TadA), which can change adenine to inosine; thus, it can convert A-T to G-C [26]. This system enables researchers to make targeted point mutations with around 50 percent efficiency. Additionally, the cytidine deaminase enzyme has been applied in manipulating several bacterial species [27-30]. This system has been designed to induce an end-codon into a gene and make it possible to out the target gene purposefully and is known as CRISPR-stop. This approach has been efficiently used to inactivate several genes in mice zygote cells [31] as well as to identify critical amino acids in proteins [32]. Also, the cytidine deaminase enzyme has been used for induction of exon skipping, activation of alternative 3' or 5' splice site, switching between mutually exclusive exons, or targeted intron retention [33]. To ease recognition sites that could be identified by the CRISPR-Cas9 system and to reduce the off-target rate, web-based software has been used. 


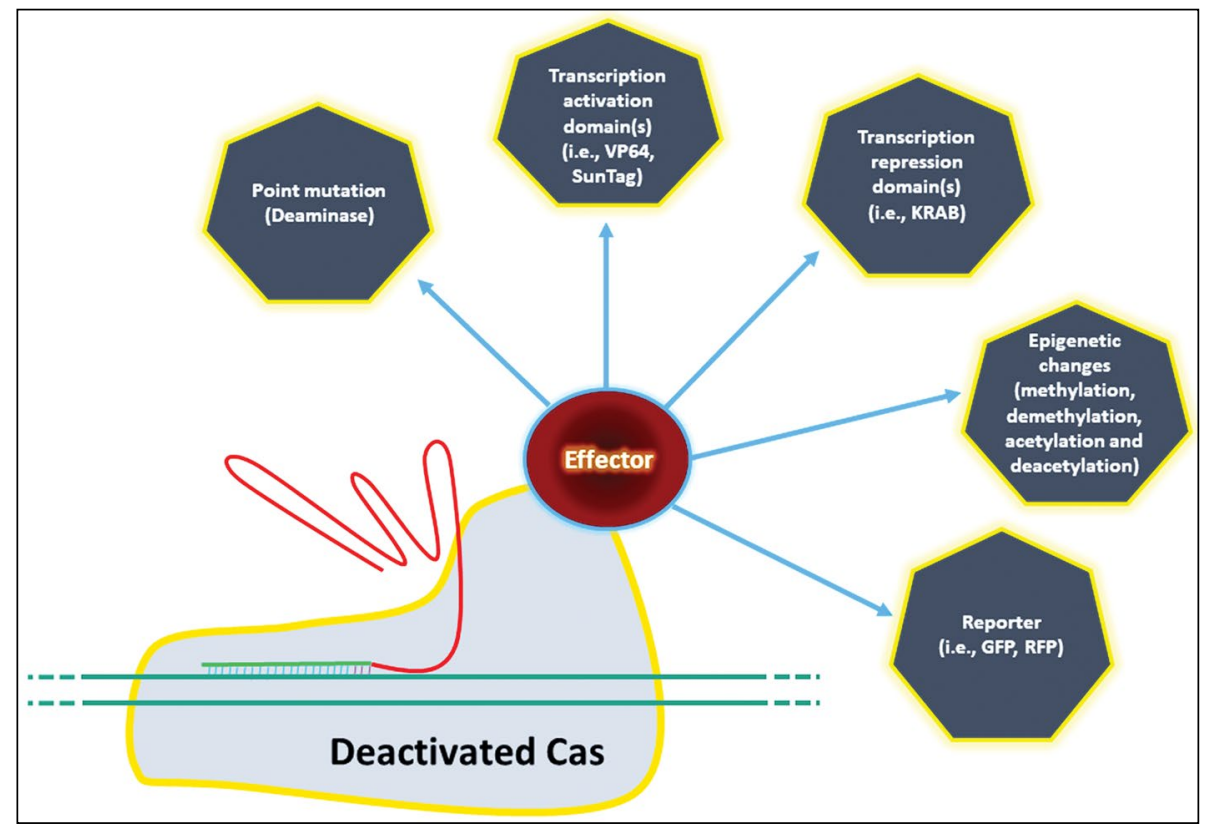

Figure 2. Researchers have mutated and inactivated Cas so that it can bind to DNA based on the sgRNA targeting sequence, but has lost its ability to cleave. By binding Cas to transcriptional activators (e.g., VP64 [Virion Protein 64], SunTag [repetitive peptide epitopes that can be used to recruit multiple copies of an antibody-fusion protein to the target of interest]) and inhibition domains (e.g., KRAB [Krüppel Associated Box]), epigenetic modulation domains, reporters (e.g., GFP [Green Fluorescent Protein], RFP [Red Fluorescent Protein]), and deaminase (i.e., adenosine deaminase), additional capabilities can be added to the Cas.

One of the major disadvantages of the CRISPR-Cas9 system is the "off-target effect". Although this system is highly efficient in prokaryotes, in the case of eukaryotes, there has been an increase in the off-target effect due to multiple increases in genome size. To overcome this effect, one specific mutation on Cas9 protein has been added to create a new protein called Cas9 nickase. This protein has a mutation in nuclease domains to cut complementary or non- complementary strand DNA with sgRNA (Fig. 2). It has been shown that using sgRNAs on opposite strands to create a double nickase resulted in 200-1500 fold reduction of off-target effect $[19,34,35]$.

\section{CRISPR-Cpf1(Cas12)}

One of the most important CRISPR-associated tools is CRISPR from Prevotella and Francisella 1 (CRISPR-Cpf1) which is analogous to the CRISPR-Cas9. Zhang and co-workers for the first time introduced the CRISPR-Cpf1 system in which the sgRNA-endonuclease protein differs from that of the CRISPR-Cas9 system [17] (Fig. 3). Crystallography of Lachnospiraceae bacterium ND2006-crRNA-DNA revealed that the Cpf1 consists of a binding domain with a looped-out helical domain (LHD), an oligonucleotide binding domain (OBD), a RuvC nuclease domain, and domains with unknown functions [36].

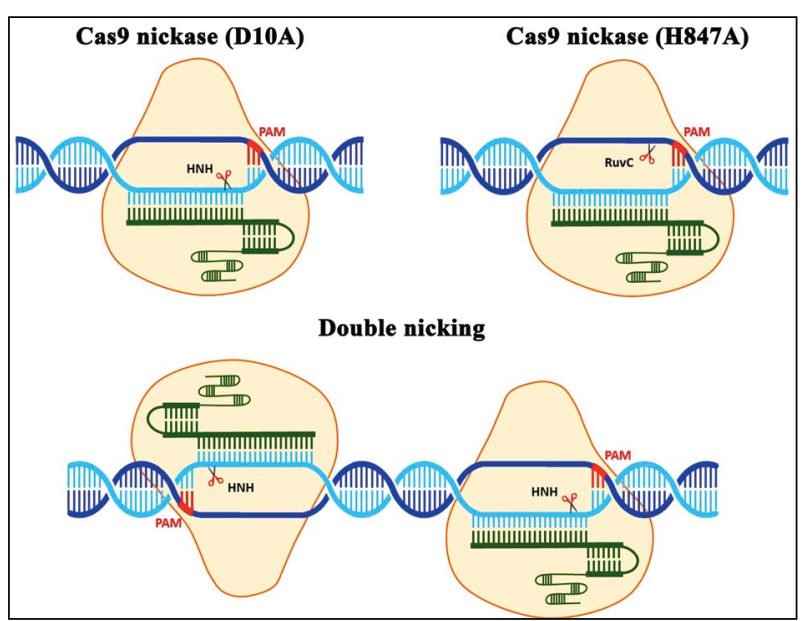

Figure 3. Single nickase and paired nickases systems. Researchers identified two amino acids in the Cas9 endonuclease protein (H840 and D10), that are involved in cutting one of the DNA strands. The amino acid H840 is located in the RuvC domain and the amino acid D10 is located in the HNH domain. Cas9 D10A cleaves the gRNA-targeting strand, while Cas9 H840A cleaves the non-targeted strand. In single nickase, the Cas9 can cut merely the strand complementary to the sgRNA; a couple of sgRNA-Cas9n complexes can nick both strands at the same time (paired nickases). When using paired nickases, additional considerations need to be given to gRNA design which includes producing a 5 ' overhang, the spacing between the two gRNAs, and the relative position of the two gRNA target sites. By using paired nickases, the off-target is greatly reduced, but cutting efficiency is also greatly reduced. 


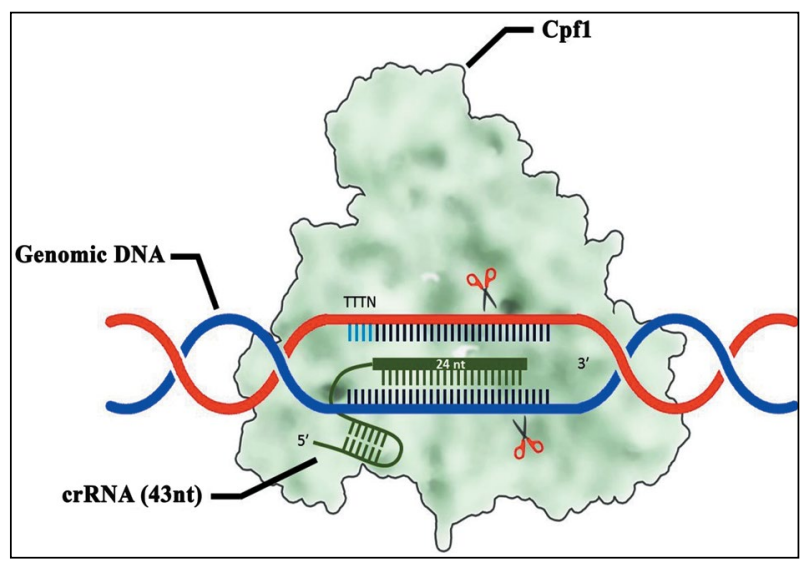

Figure 4. CRISPR-Cas12a (Cpf1) system. The Cas12a targets AT-rich regions of the genome (indicated with light blue color), in contrast to Cas9 which targets GC-rich sequences. The backbone of the sgRNA has one stem-loop and the sgRNA is composed of 43 nucleotides, of which 24 nucleotides are complementary to the target DNA (shown in dark green color). The mature crRNA required for the Cpf1 action is shorter than the guide RNA of the Cas9. The Cpf1 endonuclease protein has fewer nucleotides than the Cas9 and carries the cut as a sticky end, and the cut area is far from the PAM. Three well-studied orthologs of the Cpf1, including AsCpf1, FnCpf1, and LbCpf1 have been applied for genome editing in eukaryotic cells. The PAM sequences of the Cpf1 family proteins differ only in the number of thymidine.

A crystallography analysis on DNA-crRNA-Acidaminococcus $s p$. has shown that conformational transitions of the PAM interacting cleft and disordered seed sequence minimize the off-target in CRISPR-Cpf1 system suggesting PAM sequence plays a critical role in the stimulation of structural rearrangements [37] (Fig. 4). Using the Cpf1 endonuclease derived from Acidaminococcus sp. BV3L6 and Lachnospiraceae ND2006 bacteria, it was found that the effect of this system on eukaryotic cells is compatible with the CRISPR-Cas9 [38]. However, functional Cpf1 does not require the tracrRNA, but crRNA is needed. This promotes genome editing because the Cpf1 is not only smaller than the Cas9 but also has a smaller sgRNA molecule. Cas12a orthologs and BhCas12b have been used as powerful gene-editing tools $[39,40]$. Catalytically inactive AsCpf1 protein in fusion with a transcription activator (dAsCpf1-VPR) was used for upregulation of targeted gene expression in mammalian cells [41]. The Cpf1 has also been effectively used for multiplex gene editing purposes [42-44]. Recently, Broughton et al. used a CRISPR-Cas12-based assay to detect SARS-CoV-2 from patient RNA, namely SARS-CoV-2 DNA Endonuclease Targeted CRISPR Trans Reporter (DETECTR) [45]. Some of the new subtypes of type-V CRISPR system including Cas12e,
Cas12f, Cas12g, Cas12h, Cas12i, and Cas12k show programmable endonuclease activity [46-49].

\section{CRISPR-Cas13}

The type VI CRISPR-Cas systems also known as CRISPR-Cas 13 only requires one Cas 13 protein and a crRNA molecule. To date, four subtypes of this system have been recognized including VI-A (Cas13a/C2c2), VI-B (Cas13b/C2c6), VI-C (Cas13c/ /C2c7), and VI-D (Cas13d) [50, 51]. The presence of two HEPEN domains that are responsible for RNA-targeted nucleolytic activity is a common feature of all subtypes, even though they differ in size and sequence [50,52]. While Cas13a is used to cut a particular RNA [51], catalytically-inactive Cas13 (dCas13) can only attach to RNA strands [53-55]. Similar to CRISPR-Cas9 which present base editing activity to revert an SNP without inducing a dsDNA break [56], RNA base editing for A-to-I edit [57] and C-to-U edit [58] were proven to be achieved by fusion of dCas13 with ADAR. These systems are used to make changes at RNA level as well as to treat diseases [59]. In contrast to other types of Cas13, Cas13d [60] and some examples of Cas13a do not require a Protospacer Flanking Sequence (PFS). Very recently, it has been shown that Cas13d can inhibit SARS-CoV-2 and influenza [61]. Among the Cas13 family, the Cas13d has fewer amino acids than others (930 amino acids). Due to the low number of Cas13 nucleotides, the insertion of this protein in vectors with a size limitation is much easier. Importantly, the Cas13d has more than $90 \%$ efficiency in cutting the RNA. These features of the Cas13d endonuclease has made it as one of the best tools for molecular biology studies.

\section{Applications of CRISPR-Cas9 in biomedical engineering}

The CRISPR-Cas system has demonstrated an incredible potential for utilization in several biomedical and biotechnological applications. In this review, we focus on some of the CRISPR applications in biomedical engineering. One of the major applications of the CRISPR-Cas system is the induction of disease models in animals become faster and cheaper than conventional methods. The creation of these models is intended to simulate human diseases in mouse; due to its genomic similarity to the human genome, the low distance between generations, and its cost-effectiveness [62-65]. However, problem with CRISPR-mediated creation of animal models is the generation of mosaic animals [66]. The frequency of mosaic animals generated via the CRISPR-Cas9 system may vary in different species [66]. Nevertheless, 
this system has provided a good opportunity to study cancer in cell and animal models [67-69].

Regarding the importance of animal studies, the CRISPR has been used for knocking out the MHC system in pigs at the genome level [70] which represents a suitable source of organs for xenotransplantation. Due to its importance in the food and biomedical industry, genetically engineered pigs have been produced by introducing CRISPR components into developing zygotes using somatic cell nuclear transfer (SCNT) [71]. Also, it has been demonstrated that resistance to Mycobacterium bovis in cows can be generated through the integration of Nramp1 (natural resistance-associated macrophage protein-1) gene by the Cas9 nickase into the bovine genome [72]. CRISPR-based genome manipulation has been successfully used in other livestock animals including goat [73-76] and sheep [77-80].

CRISPR has been used in multiplexing and simultaneously targeting several genes on multiple chromosomes and modeling complex human diseases. This system has been successfully applied in patient-derived hematopoietic stem and progenitor cells [81], liver diseases including viral hepatitis caused by hepatitis $\mathrm{B}$ virus (HBV) and hepatitis $\mathrm{C}$ virus (HCV) [82], and neurological disorders including amyotrophic lateral sclerosis (ALS) and Parkinson's disease [83]. Effective delivery of CRISPR-Cas9 to target cells is critical for therapeutic editing outcomes. Using in vivo or ex vivo methods, Cas9 can be delivered directly in the form of ribonucleoprotein (RNP) complexes or indirectly as DNA or mRNA. While in vivo methods exploit direct delivery of CRISPR-Cas components into unhealthy cells of the body, ex vivo methods can be used to introduce the CRISPR-Cas components to cells harvested from patients, before reintroducing them into the patient [84]. $E x$ vivo gene editing is favorable to achieve good efficiency and safety in CRISPR-mediated genetic manipulation of the human-induced pluripotent stem cells (hiPSCs). This strategy has been implemented for treating HIV-1 infection and acute lymphoblastic leukemia [85]. In vivo gene editing therapy is more challenging as it requires more efficient and tissue-specific in vivo delivery methods. A recent preclinical study by Maeder et al. [86] documented efficient removal of a disease-causing mutation within the intron 26 of the CEP290 gene using AAV-saCas9, in both murine and primate models of Leber congenital amaurosis type 10 .

In the context of biomedical applications, CRISPR system has been used in eliminating infectious agents such as viruses [87] and genome engineering of non-infectious microorganisms to achieve bio-based benefits [88, 89].

CRISPR-Cas9 libraries have been used in drug target discovery and such approaches offer promising results over RNA interference because of its ability to target the whole genome, complete knockdown, and remarkable reproducibility [90]. Genome-wide CRISPR library screens include CRISPR-based loss-of-function (CRISPR knock-out) for studying drug resistance [91]; CRISPR-based gene activation (CRISPRa) for studying gain-of-function [92]; and CRISPR-based gene inhibition (CRISPRi) for studying the loss of functions (Figure 4) [93]. Of these, CRISPR knock-out libraries employ original Cas9, while CRISPRa and CRISPRi libraries use dCas9 along with other elements including VP64 (activation) [94] or the Krüppel associated box (KRAB) repression (inhibition) [23]. The CRISPR-Cas screens have also been applied for assessing gene deletions linked to drug resistance. One such application of CRISPR-Cas9 involves knocking out HPRT1 leading to cells' resistance to 6-thioguanine which has been commonly used in cancer treatment [95]. Another study identified 51-long noncoding RNAs involved in modulating cancer cell growth [96].

In cancer research, the CRISPR system could improve cancer diagnostic platforms that impact hospital medicine [53, 57]. It has also been used for high-throughput screening to identify anti-cancer drugs [97]. CRISPR imaging systems using dCas9 [98-101] and dCas13 [54, 57, 101] have shown to be critical for tracking genomic content and transcriptional regulation in various tissues of healthy and ill people. In cancer immunotherapy, the CRISPR has been used to generate CAR-T cells as the CRISPR engineered $T$ cells showed high potential in treating leukemia and lymphoma [102]. PD-1 has been identified as an important target for the engineering of T-cells $[103,104]$. CRISPR technologies have been widely applied in stem cells and regenerative medicine [105-107]. In recent years, several methods of nanoparticle-mediated CRISPR/Cas9 delivery systems have been well established [108].

\section{Perspectives and future directions}

Genome editing is a modern science tool and has been considered as a promising strategy for treating several diseases at genomic level. Among genome editing tools, CRISPR-Cas is now being considered as a great interest to both scientists and clinicians. CRISPR/Cas9 system is simple, relatively cheaper and its in vitro, in vivo and ex vivo applications have been used to diagnose, cure or reduce the severity of a wide range of human diseases as well as HIV-1/ /AIDS and Kostmann disease [45, 109-113]. CRISPR technology is applicable at many biological levels and could be used for the treatment of many human 
diseases including cancers, infectious diseases, and genetic disorders. It has also been shown that Cas9 protein can activate host immunity against itself [114]. Researchers have been attempting to overcome challenges including the targeted delivery of this editing system to the target area and reduction of off-target effects $[115,116]$. The CRISPR system has an off-target limit in organisms with a large genome which can be minimized by using bioinformatics and engineering tools (e.g., double nickase approach [34]. Besides, the possible immunogenicity of SpCas9 and SaCas9 proteins [114] need to be discussed for in vivo therapy. Another challenge is the large size of some Cas9 proteins (e.g., spCas9 approximately is $160 \mathrm{kDa}$ ), which is a restriction in delivery approaches, as some viral vectors have a size limitation for integration. Ethical aspects should not be ignored when working with this system. A deep understanding of the challenges and differences between several CRISPR systems allows us to improve its performance functionality. Overall, the CRISPR-Cas system toolkits (including Cas9, Cas12, and Cas13 and their engineered derivatives) are endowed with the potential to revolutionize the field of biomedicine.

The recent developments in the CRISPR-Cas system and its vast number of biotechnological applications allowed us to study gene function and carry out translational research. In recent years, CRISPR technologies, including CRISPR-mediated genome editing, CRISPRa, and CRISPRi have been widely used in gene expression studies and genome sequence manipulation. Furthermore, these systems have been applied in stem cell engineering and regenerative medicine [105]. Although the effectiveness of the CRISPR-Cas system has been documented, safe and efficient delivery of the CRISPR components to desired cells in patients' needs to be addressed. For example, in cancer immunotherapy, the most efficient method of delivery would be utilizing nanoparticles to introduce the system's components [117]. In conclusion, the CRISPR system has created a revolution in molecular biology, biotechnology, and biomedicine which rapidly moves toward creating tremendous changes in clinical trials.

\section{Funding information}

This review article did not receive any specific grant from funding agencies in the public, commercial, or not-for-profit sectors.

\section{Conflict of interest}

The authors declare no conflict of interest, financial or otherwise.

\section{References}

1. Jiang F, Doudna JA. CRISPR-Cas9 structures and mechanisms. Annu Rev Biophys. 2017; 46: 505-529, doi: 10.1146/annurev-biophys-062215-010822, indexed in Pubmed: 28375731.

2. Ishino $\mathrm{Y}$, Shinagawa $\mathrm{H}$, Makino $\mathrm{K}$, et al. Nucleotide sequence of the iap gene, responsible for alkaline phosphatase isozyme conversion in Escherichia coli, and identification of the gene product. J Bacteriol. 1987; 169(12): 5429-5433, doi: 10.1128/ jb.169.12.5429-5433.1987, indexed in Pubmed: 3316184.

3. Nakata A, Amemura M, Makino K. Unusual nucleotide arrangement with repeated sequences in the Escherichia coli K-12 chromosome. J Bacteriol. 1989; 171(6): 3553-3556, doi: 10.1128/jb.171.6.3553-3556.1989, indexed in Pubmed: 2656660.

4. Hermans P, Van So, Bik E, et al. Insertion element IS987 from Mycobacterium bovis BCG is located in a hot-spot integration region for insertion elements in Mycobacterium tuberculosis complex strains. Infect Immun. 1991; 59(8): 2695-705, indexed in Pubmed: 1649798

5. Mojica FJ, Juez G, Rodríguez-Valera F. Transcription at different salinities of Haloferax mediterranei sequences adjacent to partially modified PstI sites. Mol Microbiol. 1993; 9(3): 613-621, doi: 10.1111/j.1365-2958.1993.tb01721.x, indexed in Pubmed: 8412707.

6. Groenen PM, Bunschoten AE, van Soolingen D, et al. Nature of DNA polymorphism in the direct repeat cluster of Mycobacterium tuberculosis; application for strain differentiation by a novel typing method. Mol Microbiol. 1993; 10(5): 1057-1065, doi: 10.1111/j.1365-2958.1993.tb00976.x, indexed in Pubmed: 7934856.

7. Mojica FJ, Díez-Villase or C, Soria E, et al. Biological significance of a family of regularly spaced repeats in the genomes of Archaea, Bacteria and mitochondria. Mol Microbiol. 2000; 36(1): 244-246, doi: 10.1046/j.1365-2958.2000.01838.x, indexed in Pubmed: 10760181.

8. She Q, Singh RK, Confalonieri F, et al. The complete genome of the crenarchaeon Sulfolobus solfataricus P2. Proc Natl Acad Sci U S A. 2001; 98(14): 7835-7840, doi: 10.1073/ pnas.141222098, indexed in Pubmed: 11427726.

9. Jansen R, Embden JD, Gaastra W, et al. Identification of genes that are associated with DNA repeats in prokaryotes. Mol Microbiol. 2002; 43(6): 1565-1575, doi: 10.1046/j.13652958.2002.02839.x, indexed in Pubmed: 11952905.

10. Storici F, Snipe JR, Chan GK, et al. Conservative repair of a chromosomal double-strand break by single-strand DNA through two steps of annealing. Mol Cell Biol. 2006; 26(20): 7645-7657, doi: 10.1128/MCB.00672-06, indexed in Pubmed: 16908537.

11. Yoshimi K, Kunihiro Y, Kaneko T, et al. ssODN-mediated knock-in with CRISPR-Cas for large genomic regions in zygotes. Nat Commun. 2016; 7: 10431, doi: 10.1038/ ncomms10431, indexed in Pubmed: 26786405.

12. Bressan RB, Dewari PS, Kalantzaki M, et al. Efficient CRIS$\mathrm{PR} / \mathrm{Cas} 9$-assisted gene targeting enables rapid and precise genetic manipulation of mammalian neural stem cells. Development. 2017; 144(4): 635-648, doi: 10.1242/dev.140855, indexed in Pubmed: 28096221.

13. Zhang F, Wen Y, Guo X. CRISPR/Cas9 for genome editing: progress, implications and challenges. Hum Mol Genet. 2014; 23(R1): R40-R46, doi: 10.1093/hmg/ddu125, indexed in Pubmed: 24651067.

14. Jiang W, Bikard D, Cox D, et al. CRISPR-assisted editing of bacterial genomes. Nat Biotechnol. 2013; 31(3): 233, doi: 10.1038/nbt.2508, indexed in Pubmed: 23360965. 
15. Kimura $P$, Nakane $T$, Ishitani $R$, et al. Molecular mechanism of CRISPR. J Appl Crystallogr. 2014; 156: 935-49.

16. Wu X, Kriz AJ, Sharp PA. Target specificity of the CRISPR-Cas9 system. Quant Biol. 2014; 2(2): 59-70, doi: 10.1007/ s40484-014-0030-x, indexed in Pubmed: 25722925.

17. Yan F, Wang W, Zhang J. CRISPR-Cas12 and Cas13: the lesser known siblings of CRISPR-Cas9. Cell Biol Toxicol. 2019; 35(6): 489-492, doi: 10.1007/s10565-019-09489-1, indexed in Pubmed: 31468291.

18. Cong Le, Ran FA, Cox D, et al. Multiplex genome engineering using CRISPR/Cas systems. Science. 2013; 339(6121): 819-823, doi: 10.1126/science.1231143, indexed in Pubmed: 23287718.

19. Ran FA, Hsu PD, Wright J, et al. Genome engineering using the CRISPR-Cas9 system. Nat Protoc. 2013; 8(11): 2281-2308, doi: 10.1038/nprot.2013.143, indexed in Pubmed: 24157548.

20. Qi LS, Larson MH, Gilbert LA, et al. Repurposing CRISPR as an RNA-guided platform for sequence-specific control of gene expression. Cell. 2013; 152(5): 1173-1183, doi: 10.1016/j. cell.2013.02.022, indexed in Pubmed: 23452860.

21. Brezgin S, Kostyusheva A, Kostyushev D, et al. Dead cas systems: types, principles, and applications. Int J Mol Sci. 2019; 20(23), doi: 10.3390/ijms20236041, indexed in Pubmed: 31801211.

22. Perez-Pinera P, Kocak DD, Vockley CM, et al. RNA-guided gene activation by CRISPR-Cas9-based transcription factors. Nat Methods. 2013; 10 (10): 973-976, doi: 10.1038 /nmeth.2600, indexed in Pubmed: 23892895.

23. Gilbert LA, Horlbeck MA, Adamson B, et al. Genome-scale CRISPR-mediated control of gene repression and activation. Cell. 2014; 159(3): 647-661, doi: 10.1016/j.cell.2014.09.029, indexed in Pubmed: 25307932.

24. Kwon DY, Zhao YT, Lamonica JM, et al. Locus-specific histone deacetylation using a synthetic CRISPR-Cas9based HDAC. Nat Commun. 2017; 8: 15315, doi: 10.1038/ ncomms15315, indexed in Pubmed: 28497787.

25. Pulecio J, Verma N, Mejía-Ramírez E, et al. CRISPR/Cas9based engineering of the epigenome. Cell Stem Cell. 2017; 21(4): 431-447, doi: 10.1016/j.stem.2017.09.006, indexed in Pubmed: 28985525.

26. Gaudelli NM, Komor AC, Rees HA, et al. Programmable base editing of $\mathrm{A} \bullet \mathrm{T}$ to $\mathrm{G} \bullet \mathrm{C}$ in genomic DNA without DNA cleavage. Nature. 2017; 551(7681): 464-471, doi: 10.1038 nature24644, indexed in Pubmed: 29160308.

27. Zhou GS, Su ZY, Cai YuR, et al. Different effects of nanophase and conventional hydroxyapatite thin films on attachment, proliferation and osteogenic differentiation of bone marrow derived mesenchymal stem cells. Biomed Mater Eng. 2007; 17(6): 387-395, indexed in Pubmed: 18032820.

28. Wang Yu, Wang S, Chen W, et al. CRISPR-Cas9 and CRISPR-assisted cytidine deaminase enable precise and efficient genome editing in Klebsiella pneumoniae. Appl Environ Microbiol. 2018; 84(23), doi: 10.1128/AEM.01834-18, indexed in Pubmed: 30217854.

29. Zhao Y, Tian J, Zheng G, et al. Multiplex genome editing using a dCas9-cytidine deaminase fusion in Streptomyces. Sci China Life Sci. 2019; 63(7): 1053-1062, doi: 10.1007/ s11427-019-1559-y.

30. Gu T, Zhao S, Pi Y, et al. Highly efficient base editing in Staphylococcus aureus using an engineered CRISPR RNA-guided cytidine deaminase. Chemical Science. 2018; 9(12): 3248-3253, doi: 10.1039/c8sc00637g.

31. Zhang He, Pan H, Zhou C, et al. Simultaneous zygotic inactivation of multiple genes in mouse through CRISPR/Cas9-me- diated base editing. Development. 2018; 145(20), doi: 10.1242/ dev.168906, indexed in Pubmed: 30275281.

32. Li Q, Li Y, Yang S, et al. CRISPR-Cas9-mediated base-editing screening in mice identifies DND1 amino acids that are critical for primordial germ cell development. Nat Cell Biol. 2018; 20(11): 1315-1325, doi: 10.1038/s41556-018-0202-4, indexed in Pubmed: 30275529.

33. Yuan J, Ma Y, Huang T, et al. Genetic modulation of RNA splicing with a CRISPR-guided cytidine deaminase. Mol Cell. 2018; 72(2): 380-394.e7, doi: 10.1016/j.molcel.2018.09.002, indexed in Pubmed: 30293782.

34. Chiang TWW, le Sage C, Larrieu D, et al. CRISPR-Cas9(D10A) nickase-based genotypic and phenotypic screening to enhance genome editing. Sci Rep. 2016; 6: 24356, doi: 10.1038/ srep24356, indexed in Pubmed: 27079678.

35. Rysenkova KD, Semina EV, Karagyaur MN, et al. CRISPR/ Cas9 nickase mediated targeting of urokinase receptor gene inhibits neuroblastoma cell proliferation. Oncotarget. 2018; 9(50): 29414-29430, doi: 10.18632/oncotarget.25647, indexed in Pubmed: 30034627.

36. Dong De, Ren K, Qiu X, et al. The crystal structure of Cpf1 in complex with CRISPR RNA. Nature. 2016; 532(7600): 522-526, doi: 10.1038/nature17944, indexed in Pubmed: 27096363.

37. Nishimasu H, Ran FA, Hsu PD, et al. Crystal structure of Cas9 in complex with guide RNA and target DNA. Cell. 2014; 156(5): 935-949, doi: 10.1016/j.cell.2014.02.001, indexed in Pubmed: 24529477.

38. Safari F, Zare K, Negahdaripour M, et al. CRISPR Cpf1 proteins: structure, function and implications for genome editing. Cell Biosci. 2019; 9: 36, doi: 10.1186/s13578-019-0298-7, indexed in Pubmed: 31086658.

39. Teng F, Li J, Cui T, et al. Enhanced mammalian genome editing by new Cas12a orthologs with optimized crRNA scaffolds. Genome Biol. 2019; 20(1): 15, doi: 10.1186/s13059-019-16208, indexed in Pubmed: 30717767.

40. Strecker J, Jones S, Koopal B, et al. Engineering of CRISPR-Cas12b for human genome editing. Nat Commun. 2019; 10(1): 212, doi: 10.1038/s41467-018-08224-4, indexed in Pubmed: 30670702 .

41. Choi J, Bae T, Byambasuren N, et al. CRISPR-Cf1 activation of endogenous BMP4 gene for osteogenic differentiation of umbilical cord-derived mesenchymal stem cells. Mol Ther Methods Clin Dev. 2020(17): 309-316, doi: 10.1016/j. omtm.2019.12.010, indexed in Pubmed: 32021879.

42. Świat MA, Dashko S, den Ri, et al. Fn Cpf1: a novel and efficient genome editing tool for Saccharomyces cerevisiae. Nucleic Acids Res. 2017; 45(21): 12585-98, doi: 10.1093/nar/ gkx1007, indexed in Pubmed: 29106617.

43. Zetsche B, Heidenreich M, Mohanraju P, et al. Multiplex gene editing by CRISPR-Cpf1 using a single crRNA array. Nat Biotechnol. 2017; 35(1): 31-34, doi: 10.1038/nbt.3737, indexed in Pubmed: 27918548.

44. Yang Z, Edwards H, Xu P. CRISPR-Cas12a/Cpf1-assisted precise, efficient and multiplexed genome-editing in . Metab Eng Commun. 2020; 10: e00112, doi: 10.1016/j.mec.2019. e00112, indexed in Pubmed: 31867213.

45. Broughton JP, Deng X, Yu G, et al. CRISPR-Cas12-based detection of SARS-CoV-2. Nat Biotechnol. 2020; 38(7): 870-874, doi: 10.1038/s41587-020-0513-4, indexed in $\mathrm{Pu}-$ bmed: 32300245 .

46. Liu J-J, Orlova N, Oakes BL, et al. CRISPR-CasX is an RNA-dominated enzyme active for human genome editing. Nature. 2019; 566(7743): 218, doi: 10.1038/s41586-019-0908-x, indexed in Pubmed: 30718774. 
47. Yan WX, Hunnewell P, Alfonse LE, et al. Functionally diverse type V CRISPR-Cas systems. Science. 2019; 363(6422): 88-91, doi: 10.1126/science.aav7271, indexed in Pubmed: 30523077.

48. Karvelis T, Bigelyte G, Young JK, et al. et al.. PAM recognition by miniature CRISPR-Cas14 triggers programmable double-stranded DNA cleavage. Nucleic Acids Res. 2020; 48(9): 516-5023, doi: 10.1093/nar/gkaa208, indexed in $\mathrm{Pu}-$ bmed: 32246713 .

49. Strecker J, Ladha A, Makarova KS, et al. RNA-guided DNA insertion with CRISPR-associated transposases. Science. 2019; 365(6448): 48-53, doi: 10.1126/science.aax9181, indexed in Pubmed: 31171706.

50. Shmakov S, Abudayyeh OO, Makarova KS, et al. Discovery and functional characterization of diverse class 2 CRISPR-Cas Systems. Mol Cell. 2015; 60(3): 385-397, doi: 10.1016/j.molcel.2015.10.008, indexed in Pubmed: 26593719.

51. Abudayyeh OO, Gootenberg JS, Konermann S, et al. C2c2 is a single-component programmable RNA-guided RNA-targeting CRISPR effector. Science. 2016; 353(6299): aaf5573, doi: 10.1126/science.aaf5573, indexed in Pubmed: 27256883.

52. Smargon AA, Cox DBT, Pyzocha NK, et al. Cas13b Is a Type VI-B CRISPR-associated RNA-guided rnase differentially regulated by accessory proteins Csx 27 and Csx28. Mol Cell. 2017; 65(4): 618-630.e7, doi: 10.1016/j.molcel.2016.12.023, indexed in Pubmed: 28065598.

53. Gootenberg JS, Abudayyeh OO, Lee JW, et al. Nucleic acid detection with CRISPR-Cas13a/C2c2. Science. 2017; 356(6336): 438-442, doi: 10.1126/science.aam9321, indexed in Pubmed: 28408723.

54. Yang LZ, Wang Y, Li SQ, et al. Dynamic imaging of RNA in living cells by CRISPR-Cas13 systems. Mol Cell. 2019; 76(6): 981-997.e7, doi: 10.1016/j.molcel.2019.10.024, indexed in Pubmed: 31757757.

55. Kaminski MM, Alcantar MA, Lape IT, et al. A CRISPR-based assay for the detection of opportunistic infections post-transplantation and for the monitoring of transplant rejection. Nat Biomed Eng. 2020; 4(6): 601-609, doi: 10.1038/s41551-0200546-5, indexed in Pubmed: 32284553.

56. Rees HA, Liu DR, Rees HA, et al. Base editing: precision chemistry on the genome and transcriptome of living cells. Nat Rev Genet. 2018; 19(12): 770-788, doi: 10.1038/s41576018-0059-1, indexed in Pubmed: 30323312.

57. Abudayyeh OO, Gootenberg JS, Essletzbichler P, et al. RNA targeting with CRISPR-Cas13. Nature. 2017; 550(7675): 280 -284, doi: 10.1038/nature24049, indexed in Pubmed: 28976959.

58. Abudayyeh OO, Gootenberg JS, Franklin B, et al. A cytosine deaminase for programmable single-base RNA editing. Science. 2019; 365(6451):382-386, doi: 10.1126/science.aax7063, indexed in Pubmed: 31296651.

59. Fry LE, Peddle CF, Barnard AR, et al. RNA editing as a therapeutic approach for retinal gene therapy requiring long coding sequences. Int J Mol Sci. 2020; 21(3), doi: 10.3390/ ijms21030777, indexed in Pubmed: 31991730.

60. Yan WX, Chong S, Zhang H, et al. Cas13d Is a compact RNA-targeting type VI CRISPR effector positively modulated by a WYL-domain-containing accessory protein. Mol Cell. 2018; 70(2): 327-339.e5, doi: 10.1016/j.molcel.2018.02.028, indexed in Pubmed: 29551514.

61. Abbott TR, Dhamdhere G, Liu Y, et al. Development of CRISPR as an antiviral strategy to combat SARS-CoV-2 and influenza. Cell. 2020; 181(4): 865-876.e12, doi: 10.1016/j. cell.2020.04.020, indexed in Pubmed: 32353252.

62. Inui M, Miyado M, Igarashi M, et al. Rapid generation of mouse models with defined point mutations by the CRISPR/
/Cas9 system. Sci Rep. 2014; 4: 5396, doi: 10.1038/srep05396, indexed in Pubmed: 24953798.

63. Chen S, Sanjana NE, Zheng K, et al. Genome-wide CRISPR screen in a mouse model of tumor growth and metastasis. Cell. 2015; 160(6): 1246-60, doi: 10.1016/j.cell.2015.02.038, indexed in Pubmed: 25748654.

64. Egorova TV, Zotova ED, Reshetov DA, et al. CRISPR/ /Cas9-generated mouse model of Duchenne muscular dystrophy recapitulating a newly identified large $430 \mathrm{~kb}$ deletion in the human gene. Dis Model Mech. 2019; 12(4), doi: 10.1242/ dmm.037655, indexed in Pubmed: 31028078.

65. Lee H, Yoon DaE, Kim K. Genome editing methods in animal models. Anim Cells Syst (Seoul). 2020; 24(1): 8-16, doi: 10.1080/19768354.2020.1726462, indexed in Pubmed: 32158611.

66. Mehravar M, Shirazi A, Nazari M, et al. Mosaicism in CRISPR/ /Cas9-mediated genome editing. Dev Biol. 2019; 445(2): 156-162, doi: 10.1016/j.ydbio.2018.10.008, indexed in Pubmed: 30359560 .

67. Maddalo D, Manchado E, Concepcion CP, et al. In vivo engineering of oncogenic chromosomal rearrangements with the CRISPR/Cas9 system. Nature. 2014; 516(7531): 423-427, doi: 10.1038/nature13902, indexed in Pubmed: 25337876.

68. Wen L, Zhao C, Song J, et al. CRISPR/Cas9-mediated TERT disruption in cancer cells. Int J Mol Sci. 2020; 21(2), doi: 10.3390/ijms21020653, indexed in Pubmed: 31963842.

69. Xu M, Weng Q, Ji J. Applications and advances of CRISPR/ /Cas9 in animal cancer model. Brief Funct Genomics. 2020; 19(3): 235-241, doi: 10.1093/bfgp/elaa002, indexed in Pubmed: 32124927.

70. Reyes LM, Estrada JL, Wang ZYu, et al. Creating class I MHC-null pigs using guide RNA and the Cas9 endonuclease. J Immunol. 2014; 193(11): 5751-5757, doi: 10.4049/jimmunol.1402059, indexed in Pubmed: 25339675.

71. Sheets TP, Park CH, Park KE, et al. Somatic cell nuclear transfer followed by CRIPSR/Cas9 microinjection results in highly efficient genome editing in cloned pigs. Int $\mathrm{J}$ Mol Sci. 2016; 17(12), doi: 10.3390/ijms17122031, indexed in Pubmed: 27918485 .

72. Gao Y, Wu H, Wang Y, et al. Single Cas9 nickase induced generation of NRAMP1 knockin cattle with reduced off-target effects. Genome Biol. 2017; 18(1): 13, doi: 10.1186/s13059016-1144-4, indexed in Pubmed: 28143571.

73. Ni W, Qiao J, Hu S, et al. Efficient gene knockout in goats using CRISPR/Cas9 system. PLoS One. 2014; 9(9): e106718, doi: 10.1371/journal.pone.0106718, indexed in Pubmed: 25188313.

74. Bharati J, Punetha M, Kumar BS, et al. Genome editing in animals: an overview. Genomics and biotechnological advances in veterinary, poultry, and fisheries. 2020: 75-104, doi: 10.1016/b978-0-12-816352-8.00003-5.

75. Niu Y, Zhao X, Zhou J, et al. Efficient generation of goats with defined point mutation (I397V) in GDF9 through CRISPR/Cas9. Reprod Fertil Dev. 2018; 30(2): 307-312, doi: 10.1071/RD17068, indexed in Pubmed: 28692815.

76. $\mathrm{LiX}$, Hao F, Hu X, et al. Generation of T $\beta 4$ knock-in Cashmere goat using CRISPR/Cas9. Int J Biol Sci. 2019; 15(8): 1743-1754, doi: 10.7150/ijbs.34820, indexed in Pubmed: 31360116.

77. Crispo M, Mulet AP, Tesson L, et al. Efficient generation of myostatin knock-out sheep using CRISPR/Cas9 technology and microinjection into zygotes. PLoS One. 2015; 10(8): e0136690, doi: 10.1371/journal.pone.0136690, indexed in Pubmed: 26305800.

78. Niu Y, Jin M, Li Y, et al. Biallelic $\beta$-carotene oxygenase 2 knockout results in yellow fat in sheep via CRISPR/Cas9. 
Anim Genet. 2017; 48(2): 242-244, doi: 10.1111/age.12515, indexed in Pubmed: 27862083.

79. Kalds P, Zhou S, Cai B, et al. Sheep and goat genome engineering: from random transgenesis to the CRISPR era. Front Genet. 2019; 10: 750, doi: 10.3389/fgene.2019.00750, indexed in Pubmed: 31552084.

80. Eaton SL, Proudfoot C, Lillico SG, et al. CRISPR/Cas9 mediated generation of an ovine model for infantile neuronal ceroid lipofuscinosis (CLN1 disease). Sci Rep. 2019; 9(1): 9891, doi: 10.1038/s41598-019-45859-9, indexed in Pubmed: 31289301.

81. Hoban MD, Cost GJ, Mendel MC, et al. Correction of the sickle cell disease mutation in human hematopoietic stem/progenitor cells. Blood. 2015; 125(17): 2597-2604, doi: 10.1182/ blood-2014-12-615948, indexed in Pubmed: 25733580.

82. Wang C, Jin H, Gao D, et al. A CRISPR screen identifies CDK7 as a therapeutic target in hepatocellular carcinoma. Cell Res. 2018; 28(6): 690-692, doi: 10.1038/s41422-0180020-z, indexed in Pubmed: 29507396.

83. Chai N, Haney MS, Couthouis J, et al. Genome-wide synthetic lethal CRISPR screen identifies FIS1 as a genetic interactor of ALS-linked C9ORF72. Brain Res. 2020; 1728: 146601, doi: 10.1016/j.brainres.2019.146601, indexed in $\mathrm{Pu}$ bmed: 31843624.

84. Acharya S, Maiti S, Chakraborty D. CRISPR-Cas9 for therapy: the challenges and ways to overcome them. Genome Engineering via CRISPR-Cas9 System. 2020: 101-110, doi: 10.1016/b978-0-12-818140-9.00009-x.

85. Xu L, Wang J, Liu Y, et al. CRISPR-Edited Stem Cells in a Patient with HIV and acute lymphocytic leukemia. N Engl J Med. 2019; 381(13): 1240-1247, doi: 10.1056/NEJMoa1817426, indexed in Pubmed: 31509667.

86. Maeder ML, Stefanidakis M, Wilson CJ, et al. Development of a gene-editing approach to restore vision loss in Leber congenital amaurosis type 10. Nat Med. 2019; 25(2): 229-233, doi: 10.1038/s41591-018-0327-9, indexed in Pubmed: 30664785.

87. Soppe JA, Lebbink RJ. Antiviral Goes Viral: Harnessing CRISPR/Cas9 to combat viruses in humans. Trends Microbiol. 2017; 25(10): 833-850, doi: 10.1016/j.tim.2017.04.005, indexed in Pubmed: 28522157.

88. Sasano Yu, Nagasawa K, Kaboli S, et al. CRISPR-PCS: a powerful new approach to inducing multiple chromosome splitting in Saccharomyces cerevisiae. Sci Rep. 2016; 6: 30278, doi: 10.1038/srep30278, indexed in Pubmed: 27530680.

89. Easmin F, Sasano Yu, Kimura S, et al. CRISPR-PCD and CRISPR-PCRep: Two novel technologies for simultaneous multiple segmental chromosomal deletion/replacement in Saccharomyces cerevisiae. J Biosci Bioeng. 2020; 129(2): 129-139, doi: 10.1016/j.jbiosc.2019.08.004, indexed in $\mathrm{Pu}-$ bmed: 31585858 .

90. Wang T, Wei JJ, Sabatini DM, et al. Genetic screens in human cells using the CRISPR-Cas9 system. Science. 2014; 343(6166): 80-84, doi: $10.1126 /$ science.1246981, indexed in Pubmed: 24336569.

91. Shalem O, Sanjana NE, Hartenian E, et al. Genome-scale CRISPR-Cas9 knockout screening in human cells. Science. 2014; 343(6166): 84-87, doi: 10.1126/science.1247005, indexed in Pubmed: 24336571.

92. Joung J, Konermann S, Gootenberg JS, et al. Genome-scale CRISPR-Cas9 knockout and transcriptional activation screening. Nat Protoc. 2017; 12(4): 828-863, doi: 10.1038/ nprot.2017.016, indexed in Pubmed: 28333914.

93. Luo Ji. CRISPR/Cas9: From genome engineering to cancer drug discovery. Trends Cancer. 2016; 2(6): 313-324, doi: 10.1016/j.trecan.2016.05.001, indexed in Pubmed: 28603775.
94. Maeder ML, Linder SJ, Cascio VM, et al. CRISPR RNA-guided activation of endogenous human genes. Nat Methods. 2013; 10(10): 977-979, doi: 10.1038/nmeth.2598, indexed in Pubmed: 23892898.

95. Smurnyy Y, Cai Mi, Wu H, et al. DNA sequencing and CRISPR-Cas9 gene editing for target validation in mammalian cells. Nat Chem Biol. 2014; 10(8): 623-625, doi: 10.1038/nchembio.1550, indexed in Pubmed: 24929529.

96. Zhu S, Li W, Liu J, et al. Genome-scale deletion screening of human long non-coding RNAs using a paired-guide RNA CRISPR-Cas9 library. Nat Biotechnol. 2016; 34(12): 1279-1286, doi: 10.1038/nbt.3715, indexed in Pubmed: 27798563.

97. Shi J, Wang E, Milazzo JP, et al. Discovery of cancer drug targets by CRISPR-Cas9 screening of protein domains. Nat Biotechnol. 2015; 33(6): 661-667, doi: 10.1038/nbt.3235, indexed in Pubmed: 25961408.

98. Nelles DA, Fang MY, O'Connell MR, et al. Programmable RNA tracking in live cells with CRISPR/Cas9. Cell. 2016; 165(2): 488-496, doi: 10.1016/j.cell.2016.02.054, indexed in Pubmed: 26997482.

99. Gu Bo, Swigut T, Spencley A, et al. Transcription-coupled changes in nuclear mobility of mammalian cis-regulatory elements. Science. 2018; 359(6379): 1050-1055, doi: 10.1126/ science.aao3136, indexed in Pubmed: 29371426.

100. Duan J, Lu G, Hong Yu, et al. Live imaging and tracking of genome regions in CRISPR/dCas9 knock-in mice. Genome Biol. 2018; 19(1): 192, doi: 10.1186/s13059-018-1530-1, indexed in Pubmed: 30409154.

101. Wang H, Nakamura M, Abbott TR, et al. CRISPR-mediated live imaging of genome editing and transcription. Science. 2019; 365(6459): 1301-1305, doi: 10.1126/science.aax7852, indexed in Pubmed: 31488703.

102. Maus MV, Grupp SA, Porter DL, et al. Antibody-modified T cells: CARs take the front seat for hematologic malignancies. Blood. 2014; 123(17): 2625-2635, doi: 10.1182/ blood-2013-11-492231, indexed in Pubmed: 24578504.

103. Cyranoski D. CRISPR gene-editing tested in a person for the first time. Nature. 2016; 539(7630): 479, doi: 10.1038/nature.2016.20988, indexed in Pubmed: 27882996.

104. Choi BD, Yu X, Castano AP, et al. CRISPR-Cas9 disruption of PD-1 enhances activity of universal EGFRvIII CAR T cells in a preclinical model of human glioblastoma. J Immunother Cancer. 2019; 7(1): 304, doi: 10.1186/s40425-019-0806-7, indexed in Pubmed: 31727131.

105. Hsu MN, Chang YH, Truong VuA, et al. CRISPR technologies for stem cell engineering and regenerative medicine. Biotechnol Adv. 2019; 37(8): 107447, doi: 10.1016/j.biotechadv.2019.107447, indexed in Pubmed: 31513841.

106. Chen G, Cheng Du, Chen B. [Development of CRISPR technology and its application in bone and cartilage tissue engineering]. Nan Fang Yi Ke Da Xue Xue Bao. 2019; 39(12): 1515-1520, doi: 10.12122/j.issn.1673-4254.2019.12.19, indexed in Pubmed: 31907146.

107. Ali Q, Malik S, Malik A, et al. Role of modern technologies in tissue engineering. Arch Neurosci. 2020; 7(1), doi: 10.5812/ans.90394.

108. Aghamiri S, Ghavidel AA, Zandsalimi F, et al. Nanoparticles-mediated CRISPR/Cas9 delivery: Applications in cancer treatment and detection. J Drug Deliv Sci Technol.; 2020: 101533

109. Baylis F, McLeod M. First-in-human Phase 1 CRISPR gene editing cancer trials: are we ready? Curr Gene Ther. 2017; 17(4): 309-319, doi: 10.2174/1566523217666171121165935, indexed in Pubmed: 29173170.

110. González-Romero E, Martínez-Valiente C, García-Ruiz $\mathrm{C}$, et al. CRISPR to fix bad blood: a new tool in basic and 
clinical hematology. Haematologica. 2019; 104(5): 881-893, doi: 10.3324/haematol.2018.211359, indexed in Pubmed: 30923099.

111. Xiao Q, Guo D, Chen S. Application of CRISPR/Cas9-based gene editing in HIV-1/AIDS therapy. Front Cell Infect Microbiol. 2019; 9: 69, doi: 10.3389/fcimb.2019.00069, indexed in Pubmed: 30968001.

112. Kostyusheva A, Brezgin S, Babin Y, et al. CRISPR-Cas Systems for Diagnosing Infectious Diseases, doi: 10.20944/preprints202002.0007.v1.

113. Pittermann E, Lachmann N, MacLean G, et al. Gene correction of reversed Kostmann disease phenotype in patient-specific induced pluripotent stem cells. Blood Adv. 2017; 1(14): 903-914, doi: 10.1182/bloodadvances.2016003798, indexed in Pubmed: 29296734.
114. Charlesworth CT, Deshpande PS, Dever DP, et al. Identification of preexisting adaptive immunity to Cas 9 proteins in humans. Nat Med. 2019; 25(2): 249-254, doi: 10.1038/s41591018-0326-x, indexed in Pubmed: 30692695.

115. Zhang XH, Tee LY, Wang XG, et al. Off-target Effects in CRISPR/Cas9-mediated genome engineering. Mol Ther Nucleic Acids. 2015; 4: e264, doi: 10.1038/mtna.2015.37, indexed in Pubmed: 26575098.

116. Lino CA, Harper JC, Carney JP, et al. Delivering CRISPR: a review of the challenges and approaches. Drug Deliv. 2018; 25(1): 1234-1257, doi: 10.1080/10717544.2018.1474964, indexed in Pubmed: 29801422.

117. Rahimi H, Salehiabar M, Charmi J, et al. Harnessing nanoparticles for the efficient delivery of the CRISPR/Cas9 system. Nano Today. 2020; 34: 100895, doi: 10.1016/j.nantod.2020.100895.

Submitted: 22 July, 2020

Accepted after reviews: 17 September, 2020 Available as AoP: 24 September, 2020 